\title{
Terres gagnées et terres perdues : conséquences environnementales de l'essor de l'agro-industrie dans un désert de piémont. Le cas de l'oasis de Virú, Pérou
}

Tierras recuperadas y tierras perdidas. Consecuencias ambientales del desarrollo de la agro-industria en el desierto costero. El caso del valle de Virú, Perú

Won lands and lost lands. Environmental consequences of the agro-industry development in a piedmont oasis. The Viru valley in Peru

\section{Anaïs Marshall}

\section{(2) OpenEdition Journals}

Édition électronique

URL : http://journals.openedition.org/bifea/1482

DOI : 10.4000/bifea. 1482

ISSN : 2076-5827

\section{Éditeur}

Institut Français d'Études Andines

\section{Édition imprimée}

Date de publication : 1 août 2011

Pagination : 375-396

ISSN : 0303-7495

Référence électronique

Anaïs Marshall, «Terres gagnées et terres perdues : conséquences environnementales de l'essor de l'agro-industrie dans un désert de piémont. Le cas de l'oasis de Virú, Pérou », Bulletin de l'Institut français d'études andines [En ligne], 40 (2) | 2011, mis en ligne le 01 février 2012, consulté le 07 novembre 2020. URL : http://journals.openedition.org/bifea/1482 ; DOI : https://doi.org/10.4000/ bifea.1482

\section{(c) (i) $\odot$}

Les contenus du Bulletin de l'Institut français d'études andines sont mis à disposition selon les termes de la licence Creative Commons Attribution - Pas d'Utilisation Commerciale - Pas de Modification 4.0 International. 


\title{
Terres gagnées et terres perdues : conséquences environnementales de l'essor de l'agro-industrie dans un désert de piémont. Le cas de l'oasis de Virú, Pérou
}

\author{
Anaïs Marshall*
}

\section{Résumé}

Depuis les années 1990, le paysage des oasis rurales de la côte péruvienne est en pleine transformation. La surface agricole des vallées ne cesse de s'étendre et de nouveaux domaines agricoles s'implantent dans les interfluves désertiques. Le discours officiel du gouvernement péruvien met l'accent sur les aspects positifs de cette mutation des espaces arides en zones agricoles rentables et de la diversité des produits agricoles péruviens offerts sur les marchés internationaux. Quels en sont les impacts environnementaux au niveau local?

Après avoir présenté le paradoxe permettant au piémont côtier désertique d'être transformé en un espace agricole compétitif, cet article présente les impacts environnementaux qui affectent les acteurs locaux dans les vallées voisines des nouveaux périmètres irrigués.

Mots clés : piémont, désert, agro-industrie, irrigation, inondation des sols, baisse de la nappe phréatique

\section{Tierras recuperadas y tierras perdidas. Consecuencias ambientales del desarrollo de la agro-industria en el desierto costero. El caso del valle de Virú, Perú}

\section{Resumen}

Desde los años 1990, el paisaje de las oasis cultivadas de la costa peruana ha cambiado radicalmente. Las tierras agrícolas de los valles viejos se han extendido sin parar y nuevas áreas de cultivo se instalan

* CRESC - EA 2356. UFR LSHS - Géographie, Université Paris 13 - 99, avenue Jean-Baptiste Clément, 93430 Villetaneuse, France, UMR 8586 PRODIG. Boursière de I'IFEA (UMIFRE 17, CNRS-MAEE) entre 2005 et 2008. E-mail: anaismarshall@gmail.com 
en los intervalles desérticos. El gobierno peruano enfatiza lo positivo de la transformación de los espacios eriazos en zonas agrícolas competitivas y de la diversidad resultante de la oferta de productos peruanos en los mercados internacionales. ¿Cuales son los impactos ambientales de tal dinámica al nivel local?

Después de delinear la paradoja que constituye lo de desarrollar una actividad agrícola competitiva en un desierto, este artículo presenta los impactos ambientales que afectan los actores locales que ocupan los valles colindantes a los nuevos perímetros de tierras regadas.

Palabras clave: costa, desierto, agro-industria, riego, inundación de terrenos, salinización, sequía

\title{
Won lands and lost lands. Environmental consequences of the agro- industry development in a piedmont oasis. The Viru valley in Peru
}

\begin{abstract}
Since the 1990, rural oasis of the Peruvian coast have changed considerably. The cultivated areas of valleys are extended more and more each day and new agricultural domains appear in old desert areas. The speeches of Peruvian government officials emphasize the positive aspects of the incorporating the arid spaces into agricultural zones and the diversity of Peruvian produce in international markets. What are the environmental impacts of this policy at the local scale?

First this article presents the paradox of the transformation of a dry environment into a competitive agricultural space. Subsequently, it presents an análisis of the environmental impact and its impact on the local peasants in the valley.
\end{abstract}

Key words: piedmont, desert, agro-industry, irrigation, flood, groundwater

Depuis les années 1990, le paysage des oasis rurales de la côte péruvienne est en pleine mutation. Les périmètres irrigués des vallées ne cessent de s'étendre et de nouveaux domaines agricoles s'implantent dans les interfluves. Le discours officiel du gouvernement péruvien met l'accent sur les aspects positifs de cette transformation des espaces arides en zones agricoles rentables et de la diversité des produits agricoles péruviens présents sur les marchés internationaux. Quels en sont les impacts environnementaux au niveau local ?

On présentera tout d'abord comment le désert de piémont est devenu support de l'agriculture d'exportation suite aux politiques nationales de gestion des ressources. Ensuite, le cas de la vallée de Virú, intégrée dans le projet national d'irrigation Chavimochic, permettra d'illustrer I'application locale de ces politiques captant les ressources au service de l'agro-industrie. Finalement, les différents facteurs provoquant l'élévation du niveau de la nappe phréatique seront exposés avant que ne soit proposée une quantification des espaces affectés dans la vallée. 
Virú, Pérou: conséquences environnementales de l'essor de l'agro-industrie dans un désert de piémont

\section{LE DÉSERT DE PIÉMONT SUPPORT DE L'AGRICULTURE D'EXPORTATION AU PÉROU}

\section{1. La région côtière : un désert de piémont}

La côte péruvienne est désertique. La définition de Demangeot \& Bernus (2001 : 18) résume bien les origines du désert côtier péruvien :

« désert parce qu'anticyclonal, côtier parce que bloqué par un obstacle orographique, froid parce que longé par un upwelling ».

Cette frange étroite s'étend sur $2000 \mathrm{~km}$ du nord au sud du pays sur une largeur variant entre 50 et $100 \mathrm{~km}$.

La plaine côtière est constituée tantôt par des plaines alluviales, tantôt par des pampas désertiques. D'un point de vue écologique, les pampas sont vierges de végétation.

Les vallées alluviales sont irriguées grâce à l'action de l'homme et elles dépendent des ressources hydriques issues des précipitations dans les Andes ou des nappes phréatiques de la Côte. Le piémont littoral bénéficie d'un climat doux et ensoleillé avec des sols riches en sédiments dans les cônes d'épandage. Ces derniers sont propices à l'essor de l'activité agricole grâce à l'apport en eau dans la mesure où les acteurs locaux ont les moyens d'y accéder et d'en tirer parti. En effet, l'implantation d'entreprises agro-industrielles implique une gestion et une politique facilitant l'accès à la ressource. La Costa, grâce à son climat perpétuellement tiède et son alimentation en eaux des Andes, a été désignée par les experts et les dirigeants politiques comme la région devant permettre de développer l'agriculture d'exportation (Mesclier, 2006 : 189).

Ce développement est possible lorsque les ressources hydrique et foncière sont accessibles légalement et financièrement. Pour cela, le cadre législatif des politiques d'irrigation et des politiques foncières est passé d'une gestion collective suite à la réforme agraire, à une politique d'ouverture des marchés économiques et fonciers depuis les années 1990. Cette évolution rapide a permis d'atteindre des résultats compétitifs permettant aux productions agricoles péruviennes d'atteindre les marchés internationaux.

\section{2. Politiques nationales favorisant le développement agro- industriel dans le désert}

Un rapide rappel historique de la législation concernant la ressource hydrique permet de mieux comprendre l'organisation actuelle, et démontre comment les politiques d'irrigation évoluent suivant le gouvernement en place.

«Les eaux, sans exception aucune, sont la propriété de l'État, et son domaine est inaliénable et imprescriptible. II n'y a pas de propriété privée 
des eaux et pas de droits acquis sur elles. L'utilisation justifiée et rationnelle de l'eau, peut seulement être octroyée en harmonie avec l'intérêt social et le développement du pays $\gg 1$.

Voici le premier article du Codigo de Aguas, n 17752 du 24 juillet 1969 qui met fin à celui de 1902 de conception latifundiste (Oré, 1998 : 289). À cette époque toutes les eaux appartenaient aux propriétaires de la parcelle dans laquelle elles se trouvaient ou prenaient leur source (Del Castillo \& Castillo, 2004 : 18). Les propriétaires des haciendas avaient ainsi un pouvoir local très important.

À partir de 1969, l'État devient propriétaire de l'eau et l'administre. De nouveaux organismes sont créés : la direction des eaux et les administrations techniques d'irrigation-ATDR2 qui ont pour fonction la distribution de l'eau, gérée par des fonctionnaires de l'État.

À partir de 1979, une nouvelle organisation des utilisateurs se met en place. Des institutions autonomes - les Juntas de Usuarios JU3 et les Comisiones de Regantes $\mathrm{CR}_{4}$ — sont créées. Les utilisateurs participent alors à la gestion et à la distribution de la ressource. L'implication des petits agriculteurs reste cependant formelle dans le sens où ils achètent l'eau qui leur est ensuite distribuée selon différents critères et notamment de choix de cultures. Les agriculteurs moyens ont, quant à eux, un pouvoir important sur la gestion de l'eau car ils sont économiquement plus forts grâce à leur performance en terme de production. La parcellisation des coopératives dans les années 1980 engendre l'augmentation considérable du nombre d'irrigants ce qui fragilise notamment les ATDR par la forte croissance de la demande en eau.

La crise économique des années 1990 modifie à nouveau la gestion de la ressource. La libéralisation des marchés et le retrait de l'État se traduisent par la responsabilité grandissante des assemblées d'utilisateurs (JU). Ces dernières assurent le contrôle et l'administration de l'irrigation (Oré, 1998 : 290) et elles mettent en place un nouveau règlement du tarif de l'eau. La loi 653 de promotion des investissements renforce le transfert de la gestion aux associations, en introduisant de nouveaux éléments qui garantissent l'attribution de l'eau à ceux qui ont investi dans le forage de puits, et en créant des autorités autonomes de bassins hydrographiques (Eguren, 2004 : 48). Cette même loi transfère à I'Institut National de Développement (Inade), la charge des grands projets hydrauliques à échelle nationale et la supervision des études et des travaux financés par l'État.

Dès 1993, I'Inade est autorisée à concéder au secteur privé la construction et I'entretien de la principale infrastructure hydraulique des grands projets

1 Article $1^{\text {er }}$ de la loi générale des eaux $\mathrm{n}^{\circ} 17752$ : «Las aguas, sin excepción alguna, son de propiedad del Estado, y su dominio es inalienable e imprescriptible. No hay propiedad privada de las aguas ni derechos adquiridos sobre ellas. El uso justificado y racional del agua, sólo puede ser otorgado en armonía con el interés social y el desarrollo del país». Traduction propre.

2 ATDR signifie «administración técnica de distritos de riego».

3 Littéralement les assemblées d'utilisateurs.

4 Littéralement commissions d'irrigants. 
d'irrigation. L'entreprise sélectionnée peut ensuite amortir son investissement et faire payer ses services par les utilisateurs bénéficiaires.

Le processus de décentralisation mis en place au Pérou en 2003 se traduit par le transfert de la gestion de l'eau aux gouvernements régionaux5. Plusieurs compétences de l'État leur sont dévolues comme, entre autres, I'agriculture, I'agroindustrie, l'environnement et les ressources naturelles (Del Castillo \& Castillo, 2004 : 36). Concernant la gestion de l'irrigation, le transfert de plusieurs projets administrés par l'Inade aux gouvernements régionaux est décidé pour des raisons politiques et notamment en raison de la complexité qu'impliquent la conservation et l'entretien des grands ouvrages d'irrigation (Del Castillo \& Castillo, 2004 : 37).

L'inégale répartition de la ressource et son accès difficile engendrent des tensions sociales. Pour y faire face, les gouvernements en place souhaitent élaborer de nouvelles lois favorisant l'accès et la distribution de la ressource hydrique. De nombreux projets ont été réalisés depuis 1993. Au départ, les textes prônaient I'idée de privatisation de la ressource. L'opposition des utilisateurs n'a jamais permis au gouvernement de l'ex Président A. Fujimori de faire adopter cette loi. En 1996, une nouvelle version a été imaginée. Elle ne mentionnait plus la privatisation de la ressource mais sa concession avec l'inscription au registre de la propriété. Ce choix traduit le principe de la désolidarisation entre la propriété de la terre et I'accès à I'eau. Del Castillo \& Castillo (2004 : 50-52) expliquent que, depuis 2001, un travail important de réflexions communes, de participations des usagers et de réunions locales a permis d'avancer sur les propositions de lois. Les auteurs précisent par ailleurs que le but de cette nouvelle loi, n'est pas d'ajouter un code de plus mais d'élaborer collectivement un texte en trouvant un consensus permettant une meilleure mise en pratique des règlements (Del Castillo \& Castillo, 2004 : 52) et l'établissement d'une gouvernance autour de la ressource hydrique.

C'est finalement en 2009 qu'une nouvelle loi est promulguée. Le 13 mars, I'autorité nationale de l'eau — Autoridad nacional de agua — I'ANA est créé. Cet organisme décentralisé de l'État exerce l'administration exclusive des eaux et devient l'entité technico-normative du système national de gestion des ressources hydriques (Article 14). Le 31 mars la loi 29338 de ressources hydriques remplace la loi de 1969. À présent, I'eau constitue un patrimoine de la Nation (Article 2). L'article deux stipule qu'il n'y a pas de propriété privée sur l'eau. L'objectif est à présent la gestion intégrée de la ressource à l'échelle nationale. La gestion locale se fait à l'échelle du bassin versant.

L'historique de la législation de l'eau au cours de ces 40 dernières années est le reflet des politiques menées successivement (Castillo \& Castillo, 2004 ; Oré \& Rap, 2011). Depuis les années 1990, la gestion de l'eau est transférée aux institutions locales ce qui engendre, selon les pouvoirs locaux, des inégalités de distribution et d'administration. En effet, les choix politiques des administrateurs des grands projets d'irrigation favorisent certains agriculteurs plutôt que d'autres. La récente loi sur l'eau aura de fait des incidences locales sur la gestion de la ressource et sur les sociétés locales. 


\section{CHAVIMOCHIC : PROJET D'IRRIGATION NATIONAL EN PLEINE EXPANSION}

\section{1. Les ressources - eau et terre - au service de l'agro-industrie}

Le projet spécial Chavimochic6 $(\mathrm{PECH})$ est un projet étatique situé dans le département de La Libertad, au nord du Pérou. Le fleuve Santa se déverse originellement à la frontière entre les départements d'Ancash et de La Libertad (fig. 1). Ce projet consiste à dévier les eaux du Santa par un canal qui s'étend du sud-est au nord-ouest le long de la côte à partir de la prise d'eau, jusqu'à la vallée de Moche traversant celle de Chao et Virú, sur une longueur de $152 \mathrm{~km}$. Le canal devrait être prolongé de $133 \mathrm{~km}$ jusqu'à Chicama et atteindre une longueur de $285 \mathrm{~km}$. L'objectif agricole de ce projet est d'étendre les périmètres irrigués de 93000 à 132000 ha, avec l'amélioration de l'irrigation des terres déjà cultivées et la création de 39000 ha de terres arables dans les zones désertiques.

L'évolution du projet met en avant les différents axes des politiques nationales. Il a été impulsé au cours du premier mandat d'Alan García (1985-1990), gouvernement plus favorable à l'interventionnisme étatique, et notamment à la construction des infrastructures. Son extension agricole, avec l'implantation des entreprises agro-industrielles, s'est accélérée au cours du mandat d'A. Fujimori, favorable à la libéralisation des marchés économiques et fonciers. Le projet est institué par la loi 16667 du 27 juillet 1967. Cette dernière déclare que sa réalisation est une nécessité d'utilité publique. Le 5 septembre 1985, la direction exécutive du projet est créée par un décret suprême. Cette direction dépend alors de I'Institut National du Développement (Inade). Les ouvrages civils du projet ont débuté en 1986 au cours du premier gouvernement d'Alan García avec des fonds provenant principalement du Trésor publique (Diaz Lima, 1997 : 103). Les deux premières étapes ont été amorcées. Le gouvernement d'Alberto Fujimori (19901995) continue à financer le projet mais dans une moindre mesure. S'ajoutent alors au financement les ressources propres du projet qui proviennent notamment de la vente des terres étatiques avec un accès à une quantité spécifiée d'eau et du prix de I'utilisation de l'eau (Diaz Lima, 1997 : 103). À partir d'août 2003, suivant le processus de décentralisation, la gestion et l'administration du PECH sont transférées au Gouvernement Régional de La Libertad.

La troisième étape est l'objet de réflexions de la part du gouvernement et des institutions, gouvernementales ou non, qui prennent en considération les avantages et les inconvénients observés au cours des deux premières étapes afin d'améliorer les résultats obtenus.

L'exemple du projet Chavimochic illustre l'évolution de la gestion des ressources

- eau et terre - suite à l'ouverture des marchés. Au Pérou, les deux ressources

6 Chavimochic : nom issu des premières syllabes de chacun des quatre cours d'eau Chao, Virú, Moche et Chicama. 
Virú, Pérou: conséquences environnementales de l'essor de l'agro-industrie dans un désert de piémont

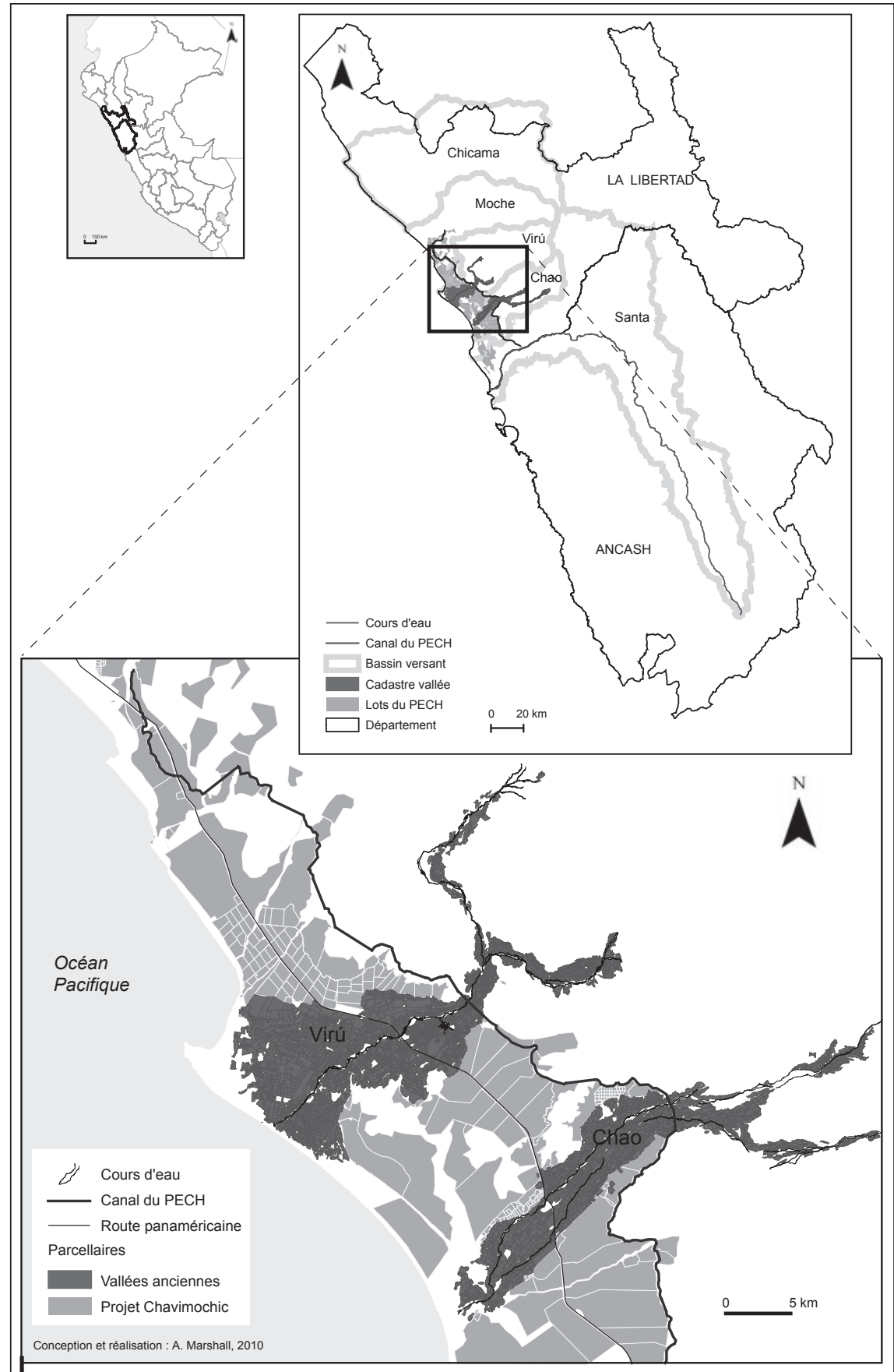

Figure 1 - Extension du projet Chavimochic en 2007 : vallées de Virú, de Chao et lots du PECH sur les interfluves

Sources: PECH, JU Virú, 2007. Conception et réalisation : A. Marshall, 2010 
sont liées : I'accès à la terre donne un droit d'accès à l'eau. Dans l'aire d'influence du $\mathrm{PECH}$, les lots des interfluves sont vendus par vente aux enchères et bénéficient automatiquement d'un droit à l'eau de 10000 m³/ha/an, qui est considérée comme la quantité minimale pour transformer le désert en zones de cultures (Pagador, 2002).

Concernant la gestion de l'eau, l'État investit et construit les infrastructures permettant l'acheminement de l'eau du Santa vers les zones de déficit hydrique. L'approvisionnement en eau depuis la rivière pérenne du Santa permet d'obtenir de l'eau dans le canal tout au long de l'année de manière relativement homogène. Au-delà des projets d'irrigation, l'État intervient dans l'administration et la distribution de la ressource hydrique. L'Institut national des ressources naturellesInrena a mission de veiller à la conservation des ressources naturelles et à leur bonne gestion. Ses services se divisent en trois ensembles dont celui de l'intendance des ressources hydriques. Ce dernier supervise la gestion et la conservation de la ressource en eau, tant superficielle que souterraine. Les eaux superficielles sont gérées localement suivant les districts d'irrigation (distritos de riego), qui regroupent plusieurs cours d'eau, et qui sont chapeautés par l'association nationale des utilisateurs des districts d'irrigation du Pérou (JNUDRP7). Sur le versant pacifique, il y a 32 districts, chacun dépendant d'une administration technique-ATDR. Cette dernière administre plusieurs JU elles-mêmes chargées de coordonner les CR, comme vu précédemment.

L'État intervient aussi dans les coûts d'utilisation des eaux superficielles. Le tarif pour l'utilisation de l'eau superficielle à des fins agricoles est explicité dans le décret suprême $n^{\circ}$ 003-90-AG. Le premier article précise que tous les usagers ont obligation de contribuer économiquement en payant le tarif de l'eau pour favoriser une utilisation rationnelle et efficiente de la ressource. Le prix de l'eau est fixé tous les ans. Le gouvernement présente, par un décret, les critères de base des prix de l'eau (Marshall, 2009 : 56). Ensuite chaque association d'irrigants fixe son coût suivant les services qu'elle apporte aux usagers.

Pour les vallées incluses dans un projet d'irrigation, les tarifs varient suivant l'origine de l'eau : cours d'eau ou infrastructures hydrauliques. Dans l'aire du PECH, il y a trois prix différents pour l'eau vendue : deux tarifs dans la vallée et un tarif pour les interfluves. En 2009, dans la vallée, ceux qui ont accès à l'eau régulée du projet, c'est-à-dire les eaux du Santa déviées par le canal, paient 0,023 sol par $\mathrm{m}^{3}$ (soit 0,007 dollars ou 0,0053 euro8) alors que ceux qui achètent l'eau du cours d'eau paient 3,5 soles pour une heure à 64 litres par seconde. Sur les interfluves, les agro-exportateurs paient 0,064 sol par $\mathrm{m}^{3}$ (soit 0,02 \$ ou 0,0148 €).

7 JNUDRP : Junta Nacional de Usuarios de los Distritos de Riego del Perú.

8 Les taux de changes sont ceux du 3e semestre 2009. 
Tableau 1 - Répartition du tarif de l'eau dans la zone du PECH en 2008 : vallées et interfluves irrigués ayant accès à l'eau déviée du Santa

Tarif élaboré suivant les différents composants listées dans le décret n003-90-AG

La différence de tarif entre les deux zones agricoles est due au prix plus élevé pour l'association locale des irrigateurs - c'est-à-dire l'association des utilisateurs d'irrigation pressurisé - qui gère la distribution de l'eau au travers d'un réseau tubulaire de haute technologie. Elle est due aussi à l'amortissement versé au PECH pour la construction du canal principal et des différentes ramifications souterraines amenant l'eau aux différents domaines agricoles.

\begin{tabular}{|c|c|c|c|c|c|c|}
\hline Zones & $\begin{array}{c}\text { Résolution } \\
\text { adminis- } \\
\text { trative }\end{array}$ & $\begin{array}{c}\text { Entrée à } \\
\text { la JU }\end{array}$ & $\begin{array}{c}\text { Redevance } \\
(10 \% \mathrm{IJU})\end{array}$ & $\begin{array}{c}\mathrm{PECH} \\
\text { amortis- } \\
\text { sement }\end{array}$ & $\begin{array}{c}\text { Part pour } \\
\text { Junta } \\
\text { Nacional } \\
\% \text { du tarif } \\
\text { total })\end{array}$ & $\begin{array}{c}\text { Tarif total } \\
\text { Soles } / \mathrm{m}^{3}\end{array}$ \\
\hline $\begin{array}{c}\text { Vallées } \\
\text { irrigués }\end{array}$ & $\begin{array}{c}\text { RA } \\
239-04-D R A- \\
\text { LL- } \\
\text { ATDRMVCH }\end{array}$ & 0,0192 & 0,00192 & 0,00192 & 0,00023 & 0,02327 \\
\hline $\begin{array}{c}\text { RA } \\
\text { Interfluves } \\
\text { irrigués }\end{array}$ & $\begin{array}{c}241-04-D R A- \\
\text { LL- } \\
\text { ATDRMVCH }\end{array}$ & 0,03103 & 0,003103 & 0,03603 & 0,00063313 & 0,06394613 \\
\hline
\end{tabular}

Les agriculteurs qui bénéficient de l'eau des projets d'irrigation paient l'entretien des infrastructures et l'accès régulier à la ressource toute l'année.

La quantité d'eau consommée par chaque agriculteur n'est pas liée à son coût. Les agro-industriels ont les moyens de payer un tarif supérieur au coût réel de la distribution de leurs besoins en eau, notamment grâce aux bénéfices financiers qu'ils obtiennent. Les petits agriculteurs quant à eux s'alimentent en eau selon les quantités provenant du cours naturel du fleuve, avec des tarifs qui diminuent lors d'excédents dus aux crues.

Par ailleurs, la variation des quantités d'eau consommée dépend davantage des systèmes d'irrigation et des cultures que du prix de la ressource. Dans la zone de Chavimochic on retrouve deux grandes techniques d'irrigation : le gravitaire et le pressurisé. Les coûts de mise en place d'un système de goutte à goutte engendrent une répartition sociale des techniques d'irrigation. Schématiquement, les entreprises agro-industrielles et les gros producteurs installent des systèmes tubulaires alors que les petits producteurs des vallées anciennes continuent à irriguer avec les systèmes gravitaire. Le développement du PECH et l'implantation des entreprises a permis à des agriculteurs petits et moyens d'accéder aux systèmes pressurisés, notamment par l'élaboration de contrats agraires entre les parties (Marshall, 2008 ; 2009).

Concernant l'accès à la terre, au niveau national, alors que la réforme agraire met en place des idéaux tel que : «la terre doit accomplir une fonction sociale » ou encore « la terre appartient à celui qui la travaille », la période néolibérale axe sa démarche sur les principes d'efficacité et de compétitivité (Eguren, 2004 : 42). Eguren (1998) explique finalement que la distribution des terres, suite à la réforme agraire, et les changements postérieurs ont diminué les grandes polarisations en 
termes de propriété. Cependant cette polarisation s'est ensuite reproduite dans la répartition et la distribution des investissements et des avancées technologiques, et elle se prolonge, sous d'autres formes.

Au niveau de l'aire d'influence du $\mathrm{PECH}$, la mise en culture de « nouvelles» terres, c'est-à-dire présentées comme désertiques et non mises en valeur par l'agriculture est un des objectifs principaux. Pour cela, des lots ont été tracés dans le désert et sont mis sur le marché suivant le système de vente aux enchères. Les investissements élevés nécessaires à la transformation de terres désertiques en terres arables nécessitent de s'orienter vers une production rentable et à forte valeur ajoutée ce qui correspond à une agriculture d'exportation. Les lots sont donc vendus à des entrepreneurs privés ou à des sociétés agro-industrielles possédant les fonds indispensables pour de tels investissements. La transaction se fait suivant deux formalités : soit par vente directe, lorsque le terrain appartient déjà à un propriétaire ou à un possesseur, soit par vente aux enchères lorsque la terre appartient à l'État. Onze ventes aux enchères ont eu lieu entre 1994 et 2005. Les dix premières ont concerné un peu moins de 31000 hectares dont 12877 ha étaient mis en culture et productifs en 2005 (PECH, 2005).

Dans la région du $\mathrm{PECH}$, les politiques nationales ont favorisé l'accès aux ressources — terre et eau - aux acteurs ayant les moyens d'investir. Pour valoriser ces terres et obtenir des bénéfices, les entreprises se spécialisent dans des productions d'exportation à forte valeur ajoutée.

\section{2. Productions pour l'exportation : exemple de I'asperge}

Les changements structurels de la politique libérale des années 1990 ont porté leurs fruits. Un nombre croissant de produits agricoles péruviens sont vendus sur les marchés internationaux. Les produits d'exportation non-traditionnels connaissent une très forte augmentation depuis la fin des années 1990. Même si certains produits étaient déjà cultivés avant, comme par exemple l'asperge, la production péruvienne connaît une diversification avec la semence de nouvelles cultures comme l'artichaut ou les oignons. Il est important de revenir sur les notions de produits d'exportation traditionnels et non traditionnels pour mieux appréhender l'évolution récente des productions agricoles péruviennes.

Les produits d'exportation traditionnels sont définis par le Congrès péruvien comme étant ceux qui, historiquement, ont constitué la plus grande part de la valeur économique des exportations9. Ils ont ainsi une valeur ajoutée moindre que

9 Produits d'exportation traditionnels, définition proposée par le Congrès péruvien: Productos de exportación que históricamente han constituido la mayor parte del valor de nuestras exportaciones. En términos relativos, tienden a tener un valor agregado menor que el de los productos no tradicionales. Legalmente, están definidos en la lista de exportaciones tradicionales del Decreto Supremo 076-92-EF. Entre ellos, se considera a los siguientes productos: algodón, azúcar, café, cobre, harina y aceite de pescado, hierro, oro, petróleo crudo y derivados, plata, plomo, zinc, etc. - tradution littérale : Produits d'exportation qui historiquement ont constitué la plus grande part de la valeur [des] exportations. En 
les produits d'exportation non-traditionnels. Il s'agit principalement du café, de la canne à sucre et du coton10. D'après la même source, les produits d'exportation non-traditionnels ont une plus forte valeur ajoutée et sont aussi appelés produits agro-industriels. On peut ainsi citer l'asperge, l'artichaut ou l'avocat.

Les oasis de la région côtière sont depuis longtemps intégrées aux marchés mondiaux. Des cultures dites traditionnelles comme la canne à sucre et le coton ont été, jusque dans les années 1990, la base des exportations agricoles. À partir des années 1990, les nouvelles denrées cultivées au Pérou s'ajoutent aux productions plus traditionnelles. Aldana et al. (2006) expliquent que l'avocat, la mangue et l'asperge connaissent un essor spectaculaire dans les plaines côtières caractérisées par la tiédeur des températures, qui bénéficient des meilleures infrastructures de communication et de la proximité des ports pour l'exportation.

La figure 2 présente les valeurs (exprimées en FOB11) des productions agricoles d'exportation traditionnelles et non-traditionnelles de 1950 à 2008. À partir de 1998, les valeurs des productions non-traditionnelles dépassent les productions d'exportation traditionnelles. La très forte croissance des 10 dernières années s'explique par les choix politiques évoqués précédemment.

L'analyse de l'évolution d'une des productions d'exportation non-traditionnelles permet de prendre conscience de la dynamique du développement. Concernant la production de la région côtière, l'asperge et ses dérivés sont les produits les

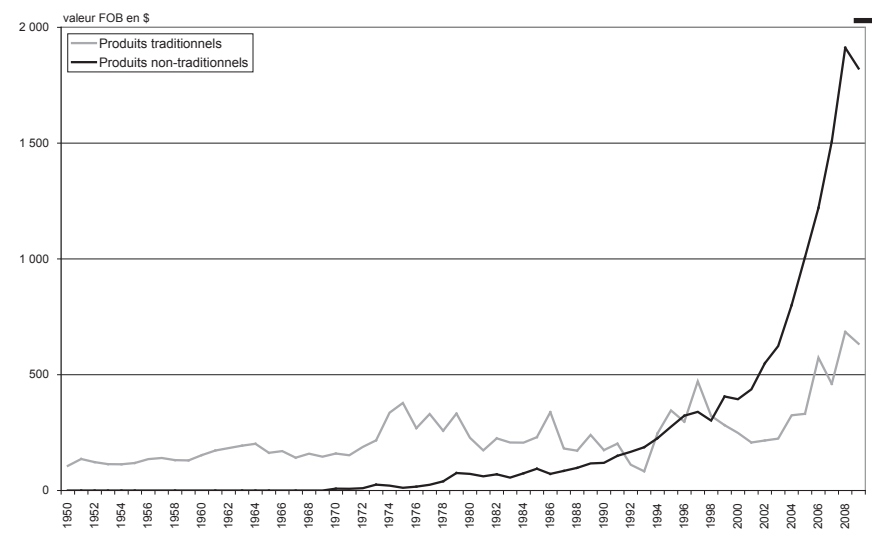

\section{Figure 2 - Exportations agricoles traditionnelles et non-traditionnelles, 1950-2008 (valeur FOB en millions de \$)}

Source : Ministère de l'Agriculture. Réalisation : A. Marshall, 2007

Les valeurs de productions d'exportation traditionnelles sont très irrégulières mais laissent transparaitre une augmentation sur la période 1950-2008. Les valeurs de productions d'exportation nontraditionnelles sont en augmentation constante avec une ascension considérable dans les années 2000.

terme relatif, elles tendent à avoir une valeur ajoutée inférieure à celle des produits non traditionnels. Légalement, [ces productions] sont définies dans la liste des exportations traditionnelles du Décret Suprême 076-92-EF. Entre autres, sont considérés les produits suivants : coton, sucre, café, cuivre, farine et huile de poisson, fer, or, pétrole brut et dérivé, argent, plomb, zinc...

10 MINAG, Ministère de l'Agriculture. www.minag.gob.pe

11 Les exportations sont indiquées en FOB. «Free On Board» est défini par la BCRP (Banco Central de Reserva del Perú) comme le terme décrivant la manière de taxer un bien quand, dans son prix, ne sont pas inclus les coûts d'exportation associés à son transfert, comme par exemple les frais de sécurité et de fret. 
plus exportés et ceux qui ont connu une très forte augmentation. Elle est produite autant dans les vallées que sur les interfluves désertiques. L'asperge est exportée fraîche (61 \%12), en conserve (31 \%) ou surgelée (8 \%). Le Pérou était, en 2006, le premier exportateur mondial d'asperges fraîches et le deuxième exportateur d'asperges en conserve.

Shimizu (2006 : 3) retrace l'évolution de cette culture du début de sa production aux années 1990. L'auteur explique que l'asperge était produite dans un premier temps dans les pays consommateurs (France, Allemagne, Hollande et États-Unis), puis la production s'est peu à peu étendue aux pays voisins (Espagne, Grèce ) pour atteindre le Pérou et la Chine grâce au déploiement des infrastructures et de la logistique.

Au Pérou, la production de l'asperge a connu trois phases successives de développement (Shimizu, 2006). Dans les années 1950, I'asperge est introduite dans la production des petits et moyens agriculteurs qui fournissent la matière première des usines. Entre 1980 et 1990, c'est I'asperge blanche pour la conserve qui connaît une forte expansion. Cette augmentation de la production est étroitement liée aux cours du marché. En effet, le Pérou et la Chine augmentent leur production alors que Taiwan, qui était le premier exportateur d'asperges blanches en conserve, se retire du marché international. Par ailleurs, les techniques sont améliorées et le système d'irrigation par goutte à goutte permet l'extension de cette culture dans le désert. Dans les années 1990, c'est I'asperge verte exportée fraîche qui connaît un fort accroissement dû à plusieurs facteurs : I'augmentation de la consommation aux États-Unis, la compétitivité des entreprises agro-industrielles et la création d'associations de certains agroindustriels (Instituto Peruano de Espárrago y hortalizas13...), la possibilité pour les producteurs péruviens de récolter toute l'année et d'atteindre ainsi des niches 14 du marché international, les accords établis notamment avec les États-Unis et I'accord ATPDEA (Andean Trade Preference and Drug Erradication Act) permettant un accès préférentiel à leur marché.

Actuellement le Pérou exporte l'asperge fraîche principalement vers les ÉtatsUnis (66 \%), puis vers la Hollande, le Royaume-Uni et l'Espagne (10 \% chacun). L'asperge en conserve est exportée principalement vers l'Espagne (29\%) puis vers la France (27\%) et le Royaume-Uni (25\%).

12 Données pour l'année 2005 (Minag - Ministerio de Agricultura).

13 Instituto Peruano de Esparragos y Hortalizas — IPEH — Institut péruvien de l'asperge et des légumes. Association sans fins lucratives formées par des agriculteurs et exportateurs d'asperges et de légumes frais ou surgelés. Sa mission est de donner les meilleurs services en terme d'innovations et de techniques aux associés et de promouvoir l'asperge et les légumes péruviens sur le marché mondial.

14 Cette notion est employée par Mesclier (2006 : 191) «les cultures pratiquées sont des produits de « niche » à haute valeur ajoutée, légumes ou fruits : asperges, artichauts, raisins de table, mangues... ». Dans notre cas, la notion de culture de niche est basée sur la définition de niche commerciale qui est un petit segment, un petit espace au sein du marché mondial ciblé en terme de clientèle et de produit, généralement nouveau et peu exploité (Petit Larousse, 2001). Plus largement, une culture de niche est ainsi une culture de faible offre ou de production rare possédant une demande importante et à haute valeur ajoutée. 
L'asperge est représentative des cultures d'exportation non-traditionnelles dans le sens où sa production fluctue selon la variation des demandes internationales. Ceci engendre une spécialisation des producteurs qui sont essentiellement des entreprises. En effet, ces derniers doivent être flexibles et avoir les moyens techniques et financiers de s'adapter aux fluctuations.

La diversification et l'augmentation des produits agricoles sur les marchés internationaux a été possible grâce à la politique d'ouverture des marchés fonciers permettant aux entreprises agro-industrielles de s'implanter et de se développer rapidement. Les conditions techniques et financières nécessaires pour s'implanter sur un espace aride, le transformer en parcelles agricoles rentables et s'adapter aux fluctuations des marchés provoquent une différenciation sociale dans l'accès à ces productions.

Au-delà de ce développement économique accessible à une partie des acteurs par des emplois directs et indirects (Marshall, 2008), quelles sont les conséquences environnementales de la modification du paysage agricole dans ces oasis ?

\section{CONSÉQUENCES ENVIRONNEMENTALES DE L'EXTENSION DE L'AGRO-INDUSTRIE}

L'apport artificiel d'eau dans le bassin versant de Virú modifie considérablement son bilan hydrique et engendre de nombreux impacts pouvant accentuer les déséquilibres naturels.

Le travail de terrain (entretiens et observations réalisés entre 2005 et 2008) a permis d'observer et de qualifier les phénomènes d'affleurement de la nappe phréatique, d'inondation des parcelles et de salinisation des sols. D'après les entretiens, l'inondation des sols vient de la déficience de l'écoulement des drains dont l'origine est anthropique et chaque groupe d'acteurs - gestionnaires et agriculteurs - rend I'autre groupe responsable des dommages. La contradiction des témoignages met en évidence le conflit qui oppose les deux groupes d'acteurs (Marshall, 2009 : 333) mais ne permet pas de déterminer les raisons du non écoulement.

Afin de valider ou non ces hypothèses, nous présenterons des facteurs anthropiques et naturels à l'origine de l'élévation du niveau de la nappe, de l'affleurement de la nappe et du risque d'inondation et de salinisation des sols, puis nous analyserons l'évolution spatio-temporelle des terres affectées par l'inondation.

\section{1. Facteurs engendrant l'élévation du niveau de la nappe}

\section{1. 1. Apport considérable de la ressource hydrique}

En 1976, Alva expliquait que sur les $40000 \mathrm{~m}^{3}$ provenant des débits de tous les cours d'eau de la région côtière, seuls 20 \% étaient utilisés à des fins agricoles (Alva et al., 1976 : 14). C'est sur le principe de récupération des eaux « perdues » dans 
I'océan que les projets d'irrigation étatiques de la côte ont été pensés et réalisés. C'est dans cette logique qu'une partie des eaux du Santa sont déviées. 27 \% sont destinées aux interfluves, $24 \%$ à la vallée de Virú, $18 \%$ à celle de Moche et $12 \%$ à celle de Chao. La majorité des eaux est ainsi déviée vers les vallées (54\%). En outre, le secteur de Virú, qui inclut la vallée et les interfluves, cumule $51 \%$ des eaux déviées du Santa.

\section{1. 2. Un débit annuel constant dans la vallée}

L'apport des eaux du Santa permet une régulation du débit des cours d'eau avec une distribution qui reste irrégulière sur l'année mais qui supprime les périodes d'étiage (Marshall, 2009).

Pour le cas de la vallée de Virú, la période de basses eaux qui naturellement dure 6 mois (de juin à novembre) n'existe plus depuis l'installation des structures hydrologiques du $\mathrm{PECH}$. Cette dernière permet ainsi de compenser les conséquences des périodes d'étiage et de sécheresse

Auparavant, pour faire face à la sécheresse, un important réseau de puits tubulaires avait été mis en place. Une étude réalisée par la FAO (Vásquez, 2000a) présente les résultats du bilan hydrique, c'est-à-dire la différence entre la valeur de la recharge et la valeur de l'extraction de la ressource en eau dans la vallée. En 1987, I'exploitation des ressources souterraines était de 71,4 millions de $\mathrm{m}^{3}$ par an avec 276 puits tubulaires et 625 à ciel ouvert. Le bilan hydrique était de moins 6 millions de mètres cubes. Cette surexploitation de la nappe au cours de la saison 19871988, a favorisé un assèchement des sols et a réduit le phénomène de salinisation naturellement présent. Lors du détournement d'une partie des eaux du Santa, le $\mathrm{PECH}$ a réalisé une campagne de sensibilisation auprès des agriculteurs pour les inciter à ne plus utiliser les eaux du sous-sol, mais celle du Santa. Chavimochic a proposé des prix de vente de l'eau inférieurs aux coûts de fonctionnement des puits. Les agriculteurs ont modifié petit à petit leurs habitudes. Vásquez (2000 : 17) explique que 98 \% des puits ne sont plus utilisés et se détériorent petit à petit.

Dans la commission de Santa Elena formée par les cultivateurs ayant droit d'arrosage et qui fait partie de la Junta de usuarios de Virú, la consommation des agriculteurs a connu une hausse de 36,6 \% (Programme IDIS15). L'apport en eau a considérablement modifié les habitudes des agriculteurs et par la même les dynamiques environnementales.

15 IDIS — Irrigation Distribution System. Système qui permet d'homogénéiser la distribution de I'eau (temps d'irrigation de la parcelle, débit attribué, horaires de début et de fin...) afin de faciliter le travail du technicien en charge de la distribution de la ressource. Il permet aussi d'informatiser toutes les données agricoles et foncières et ainsi de déterminer quelles sont les situations à régulariser. Ceci amène, petit à petit, à la formalisation des titres de propriétés du secteur. D'après le $\mathrm{PECH}$, les commissions intégrées au programme IDIS sont Huancaco, Huancaquito Bajo et Alto, Los Papayos, Santa Elena, Santa Clara et San Idelfonso ainsi que plusieurs secteurs dans la vallée de Chao. Sur le terrain, l'intégration du programme au sein de chaque commission dépend beaucoup de l'ingénieur en charge de la mise en place du système et de la disponibilité des dirigeants. 
Jusqu'en 2005, I'eau utilisée par les agriculteurs vient majoritairement du cours d'eau naturel du Virú. La tendance s'inverse à partir de 2006 où $55 \%$ des eaux distribuées proviennent du canal et 45 \% du Virú naturel $(\mathrm{PECH}, 2005)$.

\section{1. 3. Irrigation de terres anciennement désertiques}

Lors de la vente aux enchères des lots désertiques aux investisseurs, un accord est passé entre le projet d'irrigation Chavimochic et le nouveau propriétaire. Ce dernier s'engage à cultiver les lots achetés dans une période maximale de cinq années et à installer un système par goutte à goutte. De son côté, le projet s'engage à apporter un minimum de $10000 \mathrm{~m}^{3} \mathrm{~d}^{\prime}$ eau par hectare et par an.

Dans les espaces désertiques et non irrigués en 1994, c'est-à-dire sur les interfluves situés entre les vallées de Chao, Virú et Moche, I'apport en eau a atteint plus de 128 millions de $\mathrm{m}^{3}$ en $2004(\mathrm{PECH}, 2005)$. La comparaison entre les surfaces réellement cultivées et les quantités d'eau fournies permettent de calculer une consommation moyenne de $14208 \mathrm{~m}^{3}$ par hectare et par an. Au cours de la campagne agricole 2007-2008, I'apport total a dépassé les 234 millions de $\mathrm{m}^{3}$.

Les zones les plus irriguées des interfluves se situent dans les deux secteurs (III et IV) adjacents à la vallée ancienne de Virú. D'après les limites des bassins versants, les recharges naturelles de celui de Virú augmentent suite à l'irrigation de ces deux secteurs. Cet apport considérable modifie, de fait, le bilan hydrologique du bassin versant de Virú.

L'irrigation des pampas désertiques suite à la déviation d'eaux par un canal engendre un déséquilibre du budget hydrologique du bassin versant récepteur. Ces modifications anthropiques du milieu accentuent les facteurs naturels de risque d'inondation des sols. II convient à présent de les présenter.

\section{1. 4. Présence d'un ancien cordon littoral}

Le projet d'irrigation Chavimochic a construit un réseau de drains artificiels dans la vallée de Virú entre 1990 et 1992 lors de l'élaboration des infrastructures du projet. L'objectif était le drainage des sols afin d'extraire les surplus d'eau générés par l'irrigation gravitaire. Cette évacuation permet le lavage des sols afin d'éviter leur salinisation. Le service d'études du PECH avait établi un tracé théorique d'après les données physiques du milieu — topographie, pédologie, occupation du sol pour une évacuation optimum de l'eau. Dans la pratique, ce tracé n'a pas pu être réalisé car plusieurs agriculteurs ont interdit l'accès à leur terre refusant de céder plusieurs dizaines de mètres carrés sans dédommagement pour la construction des drains. Le tracé originel a ainsi été modifié et a perdu de son efficience. Le tracé actuel est réparti de façon relativement homogène dans la vallée, mais avec l'absence de drains dans la partie aval proche du littoral (fig. 3).

Les observations de terrain ont révélé que, dans certains secteurs, les drains s'écoulent dans le sens inverse, c'est-à-dire en direction des terres et non plus de la mer. Ce phénomène empêche l'évacuation des eaux et engendre le remplissage 


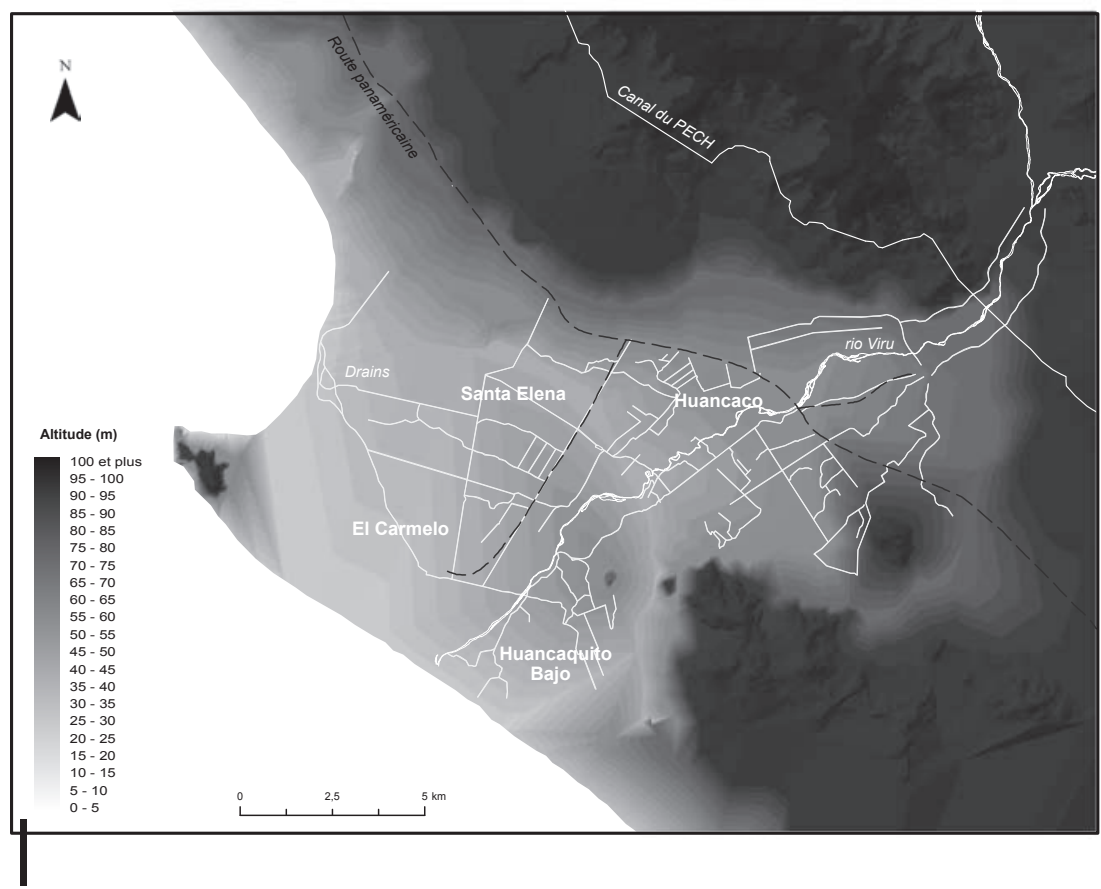

Figure 3 - Modèle numérique de terrain de la partie aval de la vallée de Virú

Source : SIG national de I'INRENA. Conception et réalisation : A. Marshall, 2008

Le MNT a été réalisé à l'aide des courbes de niveaux à intervalles de 25 mètres. Cette résolution ne permet pas de prendre en compte les variations topographiques de faibles amplitudes.

des drains de la partie centrale. La déficience de leur évacuation provoque une saturation hydrique des sols.

La réalisation et l'analyse du MNT mettent en évidence une dépression longitudinale dans la vallée de Virú. Elle se situe dans la partie moyenne de la vallée et forme un arc de cercle entre Puerto Morin et El Compositán en traversant la commission de Santa Elena. Cette dépression est un ancien trait de côte en forme d'anse dans laquelle se trouvait une île : I'actuel Cerro Negro. L'anse a été peu à peu comblée par les sédiments transportés par le cours d'eau de Virú, mais la dépression est restée. Cette dépression favorise le colmatage des drains et empêche leur écoulement vers la mer.

Par ailleurs, la proximité avec le littoral et le faible relief favorise les submersions marines à plusieurs périodes de l'année.

Les témoignages obtenus auprès des agriculteurs, des entreprises et des institutions présentent la déficience de l'écoulement des drains comme étant due à des causes anthropiques. Le travail de terrain, les entretiens avec des hydrologues et I'analyse des données topographiques complètent cette analyse en soulignant l'importance des causes naturelles, et plus spécifiquement topographiques et 
géomorphologiques avec la présence d'une dépression dans la partie centrale du delta.

Face à l'apparition de terres submergée et d'exfiltration de la nappe phréatique, nous avons souhaité quantifier ces phénomènes spatialement et temporellement.

\section{2. Mutations des espaces agricoles affectés par l'inondation}

Les observations de terrain réalisées régulièrement sur une parcelle ont permis de témoigner de l'évolution rapide des terres affectées par l'inondation. La figure 4 présente l'évolution sur un an et demi d'une parcelle de maïs inondée puis récupérée par le traçage d'un drain.

Face à ces phénomènes observés ponctuellement, nous avons souhaité quantifier spatialement et temporellement les terres inondées sur l'ensemble de la zone inondée.

Au cours des années 1999-2000, une étude réalisée par F. Vásquez, subventionnée par la FAO et supervisée par le PECH, présentait l'évolution du niveau de la nappe phréatique avant et après l'arrivée des eaux déviées du Santa (Vásquez, 2000). Cette étude de faisabilité proposait des simulations d'alternatives pour le maniement de la ressource hydrique des trois bassins versants compris dans I'aire d'influence du PECH. Elle avait pour objectif d'aider à la décision et à la bonne gestion des impacts du projet d'irrigation.

Le niveau de la nappe phréatique a ainsi été cartographié entre 1987 et 1994, soit avant la mise en place du projet, puis en 1999 après sa mise à exécution (Vasquez, 2000 ; Marshall, 2009). Cette cartographie a été réalisée grâce à un modèle mathématique, élaboré par l'École des Mines de Saint-Étienne, qui simule le comportement de la nappe phréatique.

Afin d'actualiser les données et d'obtenir des résultats comparables avec les observations réalisées lors des missions entre 2005 et 2008, nous avons réalisé une cartographie du niveau de la nappe phréatique. Ce travail s'est appuyé sur les données piézométriques de 285 puits issus de relevés réalisés par le PECH, indiquant la localisation du puit et la profondeur de la nappe. La méthode a consisté à réaliser un modèle numérique avec des données négatives correspondant à la profondeur de la nappe (Marshall, 2009).

Les nouveaux résultats ont pu être comparé à ceux de l'étude de Vásquez (2000). En 1987, la profondeur de la nappe est majoritairement supérieure à 3 mètres $(92,6 \%)$. Les profondeurs comprises entre 1 et $2 \mathrm{~m}$ puis entre 2 et $3 \mathrm{~m}$ ne correspondent chacune qu'à $3 \%$ de la superficie totale. Les profondeurs comprises entre 0 et 1 mètre correspondent à environ 128 ha c'est-à-dire moins de 1 \% de la vallée.

En 1999, la répartition est différente. Elle est plus homogène. Les profondeurs de 0 à $1 \mathrm{~m}$ ne correspondent qu'à environ 1400 ha soit $10 \%$ de la vallée. Celles de 1 à $2 \mathrm{~m}$ et de 3 et $5 \mathrm{~m}$ concernent chacune plus de $33 \%$ de la superficie. Les 
Anaïs Marshall

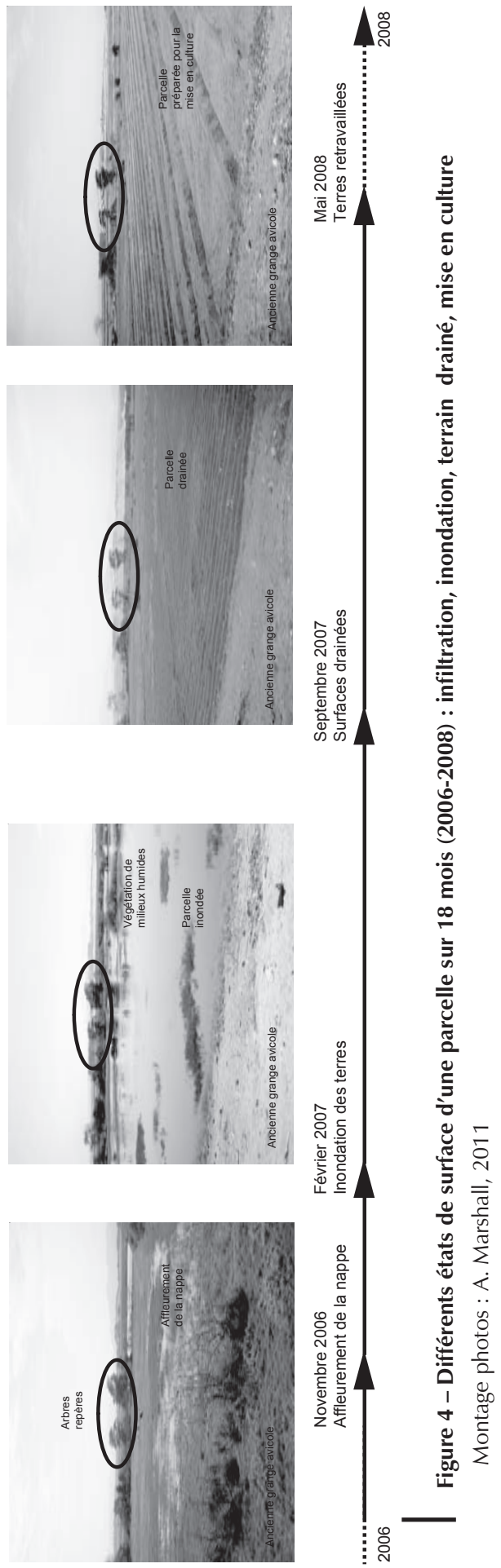


Virú, Pérou: conséquences environnementales de l'essor de l'agro-industrie dans un désert de piémont
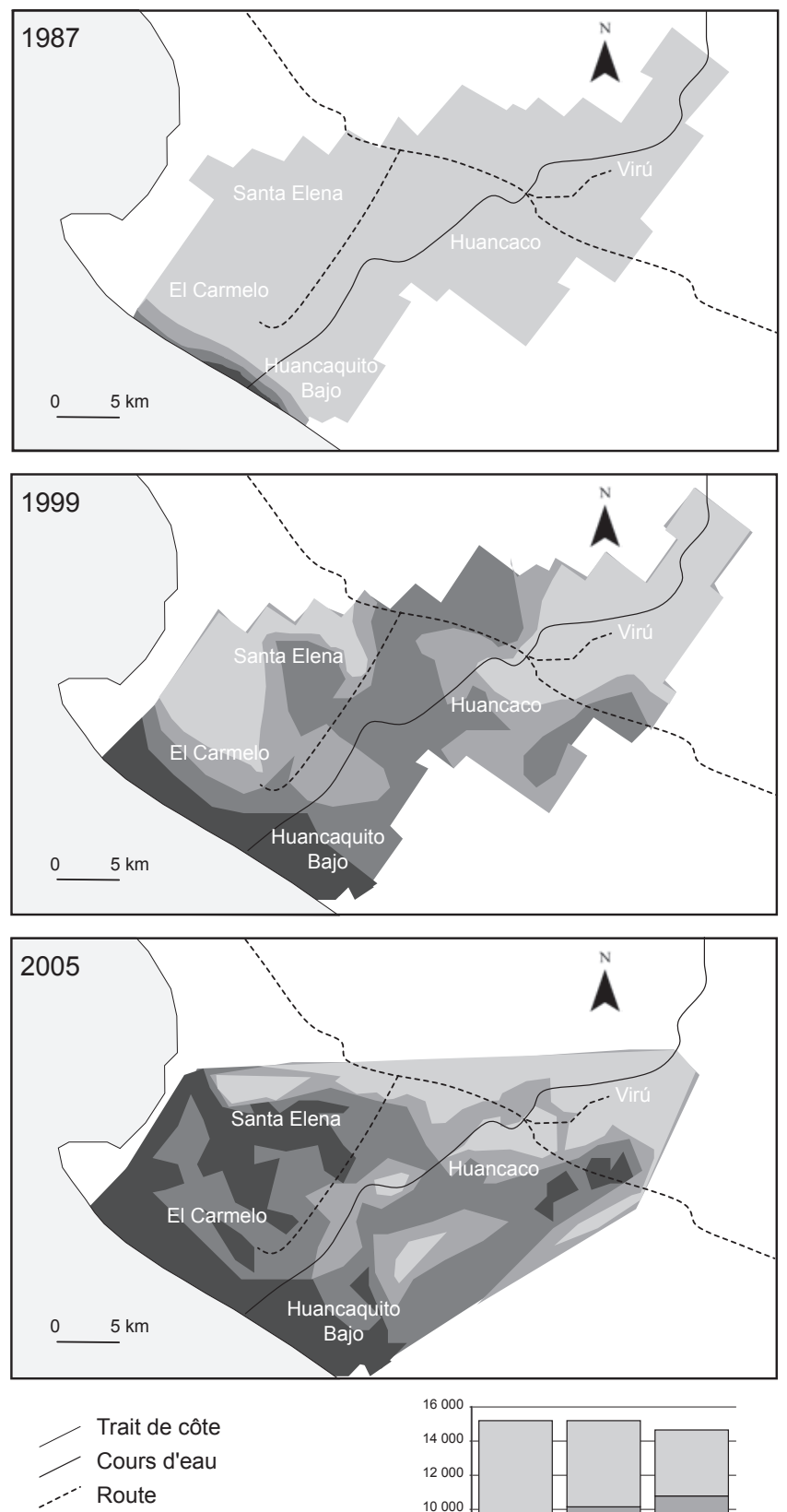

Profondeur de la nappe

Profondeur $0-1 \mathrm{~m}$

$1-2 m$

$2-3 m$

$3 \mathrm{~m}$ et plus

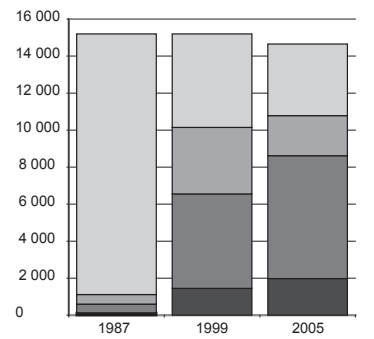

Figure 5 - Évolution de la nappe phréatique à Virú, 1987, 1999 et 2005

Source : niveaux piézométriques, $\mathrm{PECH}$ 2005. Réalisation : A. Marshall, 2011 
profondeurs de 2 à 3 m correspondent à presque 3600 ha. On observe ainsi une forte augmentation du niveau de la nappe. Les zones ayant un niveau de nappe compris entre 1 et $2 \mathrm{~m}$ ont connu une forte extension.

En 2005, la tendance s'accentue. Les superficies dont la nappe est comprise entre 0 et $1 \mathrm{~m}$ recouvrent à présent 13,5\% de la vallée, soit un peu moins de 1980 ha. Les surfaces correspondant à une profondeur comprise entre 1 et $2 \mathrm{~m}$ représentent à présent $50 \%$ de la vallée. Celles de profondeurs supérieures à $3 \mathrm{~m}$ concernent quelque 3800 ha et celles correspondant aux profondeurs comprises entre 2 et 3 m s'étendent sur 2166 ha.

La répartition de la profondeur de la nappe a considérablement évolué entre les trois années d'observation. Sur le long terme (1987-2005), on observe une tendance à l'élévation du niveau de la nappe phréatique avec une diminution des surfaces correspondant aux niveaux les plus bas. La surface de la zone comprise entre 0 et $1 \mathrm{~m}$ est passée de 129 ha à un peu moins de 1980 ha, c'est-à-dire qu'elle a été multipliée par 15. Pour les surfaces de 1 à $2 \mathrm{~m}$, la surface est passée de 468 ha en 1987 à 6638 ha en 2005. Ces deux zones représentaient ainsi moins de 4 \% de la vallée en 1987 et presque 60 \% en 2005.

Ainsi, sur la période d'analyse 1987-2005 l'élévation du niveau de la nappe apparaît clairement. Les résultats du modèle hydrologique réalisé par la FAO et le modèle numérique de profondeur de la nappe ont permis de quantifier et de localiser les espaces inondés avant la déviation des eaux du Santa en 1987, une

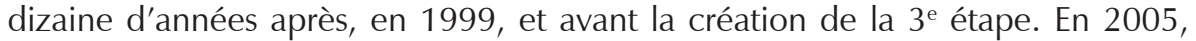
dans la région de Virú, alors que les terres gagnées sur le désert et mises en culture atteignent environ 12877 ha, les parcelles de la vallée affectées par l'élévation du niveau de la nappe et inutilisables pour l'agriculture s'étendent déjà sur plus de 8615 ha. Alors que le résultat est encore " positif », qu'en sera-t-il quand les 47000 ha vendus de la zone de Virú seront cultivées? Alors que des solutions sont pensées localement, avec notamment la réalisation ponctuelle de drains pour quelques parcelles, une gestion à l'échelle du bassin versant ou de l'aire du projet d'irrigation permettrait de réduire les conséquences environnementales qui ont un impact sur les acteurs locaux et ont créé des inégalités sociales.

\section{CONCLUSION}

Les gouvernements péruviens successifs ont su mettre en valeur la région côtière au climat doux et ensoleillé grâce à des politiques d'irrigation rendant la ressource accessible et à des politiques foncières permettant aux investisseurs de mettre en valeur les terres désertiques au profit de l'agriculture. Les productions péruviennes accèdent aux marchés internationaux et les avantages législatifs et climatologiques leur permettent d'être compétitifs.

Localement, les impacts environnementaux sont conséquents. Les grands projets d'irrigation et les modifications hydrologiques engendrées provoquent l'inondation de terres agricoles. En 2005, dans la vallée de Virú on pouvait quantifier autant de 
périmètres irrigués gagnés sur le désert que de terres agricoles perdues.

Au-delà du cas péruvien, cet exemple présente les impacts environnementaux locaux sur des territoires ruraux ouverts au processus de mondialisation. Ces espaces ruraux nouvellement attractifs connaissent d'importantes mutations notamment environnementales influençant, de fait, les sociétés locales. Finalement, la transformation des espaces et des territoires redéfinit-elle les inégalités spatiales, sociales et environnementales ou les accentue-t-elle?

\section{Références citées}

ALDANA, S., CHALÉARD, J.-L., MESCLIER, É., SALAZAR SOLER, C. \& TAYLOR, G., 2006 Le bouleversement des hiérarchies territoriales au Pérou. In : La mondialisation côté Sud. Acteurs et territoires (J. Lombard, É. Mesclier, S. Velut, eds.) : 249-272; Paris : Éditions ENS, IRD-Éditions.

ALVA, C., VAN ALPHEN J. G., TORRE DE LA, A. \& MANRIQUE, L., 1976 - Problemas de drenaje y salinidad en la Costa Peruana, 43 p. ; Wageningen, The Netherlands: Internacional Institute for Land Reclamation and Improvment/ILRI bulletin 16.

DEL CASTILLO PINTO, L. \& CASTILLO, P., 2004 - La legislación de aguas en el Perú, 60 p. ; Lima : Centro Peruano de Estudios Sociales CEPES. Informe legal agrario $n^{\circ} 20$.

DEMANGEOT, J. \& BERNUS, E., 2001 - Les milieux désertiques, 295 p. ; Paris : Armand Collin (coll U géographie).

DIAZ LIMA, C., 1997 - Política liberal y proyectos de irrigación. Revista de la Facultad de Ciencias Económicas de la San Marcos, Vol. 2, n 6 : 98-107; Lima.

EGUREN, F., 1998 - La situación de la agricultura y de la sociedad rural en el Perú ; Lima : CEPES -Secretariado Rural. Perú-Bolivia.

EGUREN, F., 2004 - Las políticas agrarias en la última década: una evaluación. In : Perú: el problema agraria en debate, SEPIA X (F. Eguren, M. I. Remy, P. Oliart, eds.) : 19-78 ; Lima: SEPIA.

MARSHALL A., 2008 - El proyecto especial Chavimochic: contratos agrarios establecidos entre agro exportadores y pequeños agricultores en los valles de Virú y Chao. In : Perú: el problema agraria en debate. SEPIA 12 : 553-584 ; Lima : Seminario Permanente de Investigación Agraria-SEPIA, Oxfam, ITDG soluciones prácticas, Consorcio de investigación económico y social, Sociedad peruana de derecho ambiental.

MARSHALL A., 2009 - S'approprier le désert. Agriculture mondialisée et dynamiques socio-environnementales sur le piémont côtier du Pérou, 493 p. ; Paris : Université de Paris 1-Panthéon-Sorbonne. Thèse de Doctorat sous la direction de Jean-Louis Chaléard.

MESCLIER, É., 2006 - Les espaces ruraux de l'Amérique latine dans la mondialisation. In : Géographies de l'Amérique latine (F. Dureau, V. Gouëset, É. Mesclier, eds.) : 139230 ; Rennes : PUR.

ORÉ, M. T., 1998 - De la reforma agraria a la privatización de tierras y aguas: el caso de la costa peruana. In : Buscando la equidad. Concepciones sobre justicia y equidad en el riego campesino (R. Boelens \& G. Dávila, eds.) : 286-297 ; Assen :Van Gorcum \& Comp. Préface de R. Menchú. 
ORÉ, M. T. \& RAP, E., 2011 - Políticas neoliberales de Agua en el Perú. Entretelones y antecedentes de la ley de recursos hídricos. Debate en Sociología, 34 : 32-65 ; Lima : PUCP.

PAGADOR, C., 2002 - Case study from Peru. Survey on irrigation modernization. Sistema de irrigation de Pur Pur, 14 p. ; Documentos de estudios de la FAO. http://www.fao. org/landandwater/aglw/watermanagement/docs/MOD_Peru.pdf

PECH, PROYECTO ESPECIAL CHAVIMOCHIC, 2005 - Chavimochic en cifras 1986-2004, 45 p.; Trujillo : Gobierno regional la Libertad, Proyecto Especial Chavimochic.

PETIT LAROUSSE ILLUSTRÉ, 2001 - 1786 p.

SHIMIZU, T., 2006 - Expansion of asparagus. Production and Exports in Perú. Institute of Developing Economies Discussion paper $n^{\circ}$ 073. http://www.ide.go.jp/English/ Publish/Download/Dp/pdf/073.pdf

VÁSQUEZ, F., 2000 - Actualización de los estudios del nivel freático en los valles de Chao, Virú y Moche, 45 p. ; FAO : proyecto TCP/PER/8823. Proyecto Especial Chavimochic. 